\title{
Crianças e adolescentes quilombolas apresentam alta prevalência de defeitos de desenvolvimento do esmalte
}

\author{
Quilombola children and adolescents show high prevalence \\ of developmental defects of enamel
}

Thalita Karenyne Xavier Silva França (https://orcid.org/0000-0002-5854-1593) ${ }^{1}$

Marina de Deus Moura de Lima (https://orcid.org/0000-0002-7641-6331) ${ }^{2}$

Cacilda Castelo Branco Lima (https://orcid.org/0000-0002-2977-6035) ${ }^{2}$

Marcoeli Silva de Moura (https://orcid.org/0000-0002-9044-9025) ${ }^{2}$

Teresinha Soares Pereira Lopes (https://orcid.org/0000-0002-1698-8725) ${ }^{3}$

Joyce Samandra Silva de Moura (https://orcid.org/0000-0001-6544-9632) ${ }^{2}$

Lúcia de Fátima Almeida de Deus Moura (https://orcid.org/0000-0002-4112-1533) ${ }^{2}$

${ }^{1}$ Instituto Federal de Educação, Ciência e

Tecnologia do Piauí. R. Álvaro Mendes 94, Centro (Sul). 64000-040 Teresina PI Brasil.

mouraiso@uol.com.br ${ }^{2}$ Programa de Pós-

Graduação em Odontologia,

Universidade Federal do

Piauí. Teresina PI Brasil.

${ }^{3}$ Departamento de Patologia

e Clínica Odontológica,

Universidade Federal do

Piauí. Teresina PI Brasil.

\begin{abstract}
Teeth with developmental defects of enamel (DDE) have porous and/or uneven enamel, making them more susceptible to the build-up of oral biofilm and development of caries and periodontal diseases. The aim of this cross-sectional study was to determine the prevalence of DDE and associated factors among children and adolescents living in a Quilombola community in the Northeast of Brazil. The study population was census-based and comprised individuals aged three to 14 years. The children's parents/guardians answered a questionnaire devised to collect information on socioeconomic and demographic characteristics, health problems during pregnancy and illnesses during early childhood. DDE was diagnosed using the modified DDE index. The data were analyzed using descriptive statistics and Poisson regression with robust standard errors $(p<0.05)$. A total of 406 individuals were examined. DDE prevalence was $80.5 \%$ : $42.2 \%$ in deciduous teeth and $61.1 \%$ in permanent teeth. There was an association between presence of DDE and age $(P R=1.09,95 \% C I=1.01-1.17)$, use of antibiotics during pregnancy $(P R=1.14,95 \% C I=1.07-1.22)$ and reported malnutrition during early childhood $(P R=1.12 ; 95 \% C I=1.03-1.22)$. The findings reveal high prevalence of DDE among children and adolescents living in the Quilombola community. Associated factors were older age, use of antibiotics during pregnancy and malnutrition during early childhood. Key words Dental enamel, Dental enamel hypoplasia, Risk groups, Cross-sectional studies
\end{abstract}

Resumo Dentes com defeitos de desenvolvimento do esmalte (DDE) apresentam porosidades e/ou irregularidades que os tornam suscetiveis a acúmulo de biofilme e predisposição à cárie e doenças periodontais. O objetivo deste artigo é determinar a prevalência de DDE e fatores associados em crianças e adolescentes residentes em comunidade quilombola. A população foi censitária e composta por indivíduos na faixa etária de 3 a 14 anos. Os responsáveis responderam a questionário contendo dados socioeconômicos demográficos e histórico de agravos durante a gravidez e infância. Foi aplicado o Índice DDE modificado. Foram realizadas análise descritiva e regressão de Poisson com variância robusta $(p<0,05)$. Foram examinados 406 indivíduos. A prevalência de DDE foi de $80,5 \%$, sendo que em dentes decíduos foi de $42,2 \%$ e permanentes $61,1 \%$. Houve associação entre DDE e maior idade da criança $(R P=1,09 ; I C 95 \%=1,01-1,17)$, uso de antibiótico na gravidez ( $R P=1,14$; IC95\%=1,071,22) e relato de desnutrição durante a primeira infância $(R P=1,12 ; I C 95 \%=1,03-1,22)$. A prevalência de DDE em crianças e adolecentes da comunidade quilombola foi alta. E os fatores associados foram maior idade da criança, uso de antibióticos na gravidez e desnutrição durante a primeira infância.

Palavras-chave Esmalte dentário, Hipoplasia do esmalte dentário, Grupos de risco, Estudos transversais 


\section{Introdução}

Quilombolas são grupos étnico-raciais segundo critérios de autoatribuição e trajetória histórica própria, áreas territoriais específicas e presunção na ancestralidade negra relacionada com resistência à opressão histórica. Apesar das políticas de inclusão social, estudos sobre saúde bucal destas populações são escassos ${ }^{1-3}$. Dentro do contexto abordado, indivíduos que residem em comunidades quilombolas apresentam acesso restrito a bens e serviços e consequente insegurança alimentar $^{1,4,5}$.

Pessoas que vivem em vulnerabilidade social apresentam em geral deficiência de nutrientes essenciais na dieta, condição que tem sido apontada como fator associado a defeitos de desenvolvimento do esmalte dentário (DDE) ${ }^{6-11}$. Essas alterações dentárias manifestam-se clinicamente como opacidades ou hipoplasias ${ }^{12-14}$. Dentes com DDE apresentam esmalte poroso e/ou irregular, condições que os tornam mais suscetíveis a acúmulo de biofilme dental e consequente predisposição ao desenvolvimento de cárie dentária e doenças periodontais ${ }^{7,14-17}$.

A etiologia de DDE é multifatorial, podendo estar associada a condições sistêmicas ou ambientais que ocorreram durante o período de amelogênese $\mathrm{e}^{6,14,15}$. Estudos epidemiológicos sobre prevalência e fatores associados ao DDE em populações vulneráveis socialmente como quilombolas, são importantes para o planejamento de políticas públicas de saúde com implementação de projetos de promoção da saúde direcionados às gestantes e crianças em idade pré-escolar, tendo em vista que esses defeitos estão relacionados a alterações durante a gravidez, primeira infância e nutrição inadequada. Este é o primeiro estudo de base populacional que objetivou avaliar a prevalência de DDE e fatores associados em comunidades quilombolas

\section{Métodos}

Trata-se de estudo transversal realizado na comunidade quilombola rural Lagoas, localizada a 36 quilômetros do município de São Raimundo Nonato, na região Sul do estado do Piauí. A comunidade é formada por 12 núcleos que abrangem 1.498 famílias e 5.128 indivíduos, distribuídos em território de 62.000 metros quadrados. Este estudo foi conduzido seguindo as diretrizes do STROBE Statement.

De acordo com dados do Instituto Nacional de Colonização e Reforma Agrária (INCRA) ${ }^{18}$, em 2010 havia 420 crianças e adolescentes quilombola na comunidade. A população deste estudo foi censitária e constituída por crianças e adolescentes de ambos os sexos na faixa etária de 3 a 14 anos. Foram excluídos do estudo indivíduos que apresentavam amelogênese imperfeita, lesões cariosas com mais de $2 / 3$ de comprometimento do esmalte ou usavam aparelho ortodôntico fixo, condições que dificultam diagnóstico de DDE.

Como estratégia para recrutamento dos indivíduos, as escolas $(\mathrm{N}=4)$ foram utilizadas como referência. Quanto às crianças que ainda não frequentavam escolas, foi realizada busca ativa por intermédio de líderes comunitários, junto aos pais/responsáveis para que levassem seus filhos no dia dos exames agendados para os escolares.

Previamente à realização do estudo, foi realizada calibração de três examinadoras em duas fases. A primeira fase compreendeu treinamento in lux ${ }^{19} \mathrm{com}$ projeções de imagens de dentes com diferentes tipos de defeitos de esmalte. Quando os examinadores deveriam acertar mais de $80 \%$ dos diagnósticos para permanecer na pesquisa. A segunda fase de treinamento foi realizada com 45 escolares, não participantes da pesquisa, que apresentavam todas as condições que seriam observadas no estudo. Os indivíduos foram reexaminados após duas semanas. As concordâncias intraexaminadores e interexaminadores foram iguais ou maiores que $0,8^{19}$.

Foi realizado estudo piloto com 45 crianças e adolescentes, estudantes de escola pública rural do município de São Raimundo Nonato, Piauí, que não foram incluídos na pesquisa. Não houve necessidade de adequações da metodologia proposta.

A coleta de dados constou de duas fases. Aplicação de questionário aos pais/responsáveis que abordava dados demográficos e socioeconômicos (sexo, idade, renda familiar, escolaridade dos pais, condições sanitárias e hábitos alimentares) e fatores citados em outros estudos como possivelmente $^{8,13,20}$ associados a DDE (tipo de parto, doenças na gravidez e primeira infância) e exame dentário das crianças e adolescentes. Os questionários foram aplicados pelos pesquisadores diretamente aos pais, de forma individualizada, para que pudessem esclarecer eventuais dúvidas que pudessem surgir no momento do preenchimento.

Os exames dentários foram realizados sob luz artificial, fluorescentes de teto. Os participantes da pesquisa foram posicionados sentados em cadeiras ou bancos escolares com a cabeça apoiada sobre as pernas dos examinadores. Para o exame, os pesquisadores utilizaram espelho bucal plano, 
sonda exploradora de número 5 (SSWhite, Rio de Janeiro, Brasil), compressas de gaze para remover o excesso de saliva.

Os dados foram anotados em ficha elaborada para este estudo. O diagnóstico de DDE foi realizado pelo índice DDE modificado ${ }^{21}$, que avalia a presença dos defeitos e suas combinações, localização e extensão. Foram classificados como opacidades os defeitos qualitativos envolvendo alteração na translucidez do esmalte, como opacidade demarcada (quando apresentava limite definido com o esmalte adjacente normal) e opacidade difusa, (quando não era observado limite definido com o esmalte normal). Foram classificados como hipoplasias, defeitos quantitativos associados à redução localizada de espessura de esmalte. Em relação à localização, os defeitos foram classificadas em 1/2 incisal, 1/2 gengival, 1/2 oclusal e em cúspide. E quanto à extensão, foram classificados em menos de $1 / 3$ da superfície, mais de $1 / 3$ e menos de $2 / 3$ da superfície, e mais de $2 / 3$ da superfície ${ }^{21}$.

Não foram incluidos no estudo dentes que apresentavam fraturas coronárias, dentes que apresentavam lesões cariosas e/ou restaurações extensas (envolvendo mais de dois terços da superfície) e dentes decíduos extraídos ou esfoliados. Defeitos unitários medindo menos de um milímetro de diâmetro não foram registrados e em casos dúbios, o defeito não foi considerado ${ }^{21}$.

Os dados foram analisados pelo programa Statistical Package for the Social Science (SPSS ${ }^{\circledR}$ para Windows, versão 21.0, Armonk, NY, USA: IBM Corp). A variável dependente foi dicotomizada quanto presença ou não de DDE. As variáveis independentes foram sexo, idade, autodeclaração da cor da pele, dados socioeconômicos como renda familiar e escolaridade da mãe, condições sanitárias, intercorrências durante a gravidez (febre ou infecção, hipertensão, infecção urinária, uso de antibióticos, sangramento, descolamento prematuro da placenta e eclâmpsia) e doenças durante a primeira infância (diarreia, varicela, pneumonia, asma, bronquite, sinusite, rinite, febre, desnutrição, otite e uso de antibióticos) $)^{1,4-7,20}$.

Foi realizada análise descritiva para caracterizar a amostra. Regressão de Poisson com variância robusta foi utilizada para determinar as associações entre DDE, como desfecho binário, e as variáveis independentes. A magnitude da associação foi avaliada pelas razões de prevalência brutas e ajustadas (RP), intervalos de confiança
(IC 95\%), e valores de p. As variáveis com os valores de $\mathrm{p} \leq 0,20$ na análise bivariada foram incluídas no modelo ajustado. Apenas as variáveis com valor de $\mathrm{p}<0,05$ permaneceram no modelo final.

Este estudo foi aprovado pelo Comitê de Ética em Pesquisa da Universidade Federal do Piauí e obedeceu aos preceitos determinados pela Declaração de Helsinque e Resolução 466/12 do Conselho Nacional de Saúde (CNS).

\section{Resultados}

Foram examinadas 406 (96,9\%) crianças ou adolescentes que preenchiam os critérios de inclusão e não houve recusas em participar do estudo. As perdas ocorreram devido ao uso de aparelho ortodôntico fixo por cinco adolescentes, e quatro apresentavam amelogênese imperfeita e cinco lesões cariosas comprometendo mais de $2 / 3$ de esmalte.

A média de idade foi de $8,74( \pm 3,17)$ anos (mínima de 3 anos e máxima de 14 anos), sendo $52,0 \%$ do sexo masculino e $35,7 \%$ autodeclarados negros. A maioria das famílias apresentou renda familiar menor ou igual a $\mathrm{R} \$ 500,00(82,5 \%)$. O salário mínimo vigente na época do estudo correspondia a $\mathrm{R} \$ 880,00$ (valor do dólar $\mathrm{R} \$ 3,2403$ ), e escolaridade materna menor que oito anos de estudo formal (49,5\%). A água utilizada nos domicílios provinha de cisternas abastecidas por carros-pipa $(78,6 \%)$, tratada com a adição de tabletes efervescentes de cloro (41,1\%) (Tabela 1).

A prevalência de DDE foi de 80,5\% (Tabela 1), sendo que em dentes decíduos foi de $42,2 \%$ e permanentes $61,1 \%$. O tipo de defeito mais frequente em ambas as dentições foi opacidade demarcada. As áreas dos dentes mais afetadas foram metade incisal e ponta de cúspide. A maioria das crianças e adolescentes apresentou defeitos de esmalte em menos de $1 / 3$ de superfícies dentárias (Tabela 2).

Os defeitos de desenvolvimento do esmalte foram 9,0\% $(\mathrm{RP}=1,09$; IC95\%=1,01-1,17) mais prevalentes na faixa etária de 11 a 14 anos em relação às crianças de 3 a 5 anos. Entre as intercorrências durante a gravidez, uso de antibiótico $(\mathrm{RP}=1,14$; IC95\%=1,07-1,22) foi associado à presença de DDE. O relato dos pais/responsáveis sobre desnutrição durante a primeira infância $(\mathrm{RP}=1,12$; $\mathrm{IC} 95 \%=1,03-1,22)$ foi associada à presença de DDE (Tabela 3 ). 
Tabela 1. Perfil socioeconômico e demográfico das crianças e adolescentes residentes na comunidade quilombola Lagoas, Sul do estado do Piauí.

\begin{tabular}{|c|c|c|c|}
\hline \multirow[t]{2}{*}{ Variáveis } & \multirow[t]{2}{*}{$\mathrm{N}(\%)$} & \multicolumn{2}{|c|}{$\begin{array}{c}\text { Defeitos de } \\
\text { Desenvolvimento } \\
\text { do Esmalte }\end{array}$} \\
\hline & & $\begin{array}{c}\text { Presença } \\
\text { N (\%) }\end{array}$ & $\begin{array}{c}\text { Ausência } \\
\text { N (\%) }\end{array}$ \\
\hline \multicolumn{4}{|l|}{ Idade (anos) } \\
\hline 3 a 5 & $79(19,5)$ & $56(70,9)$ & $23(29,1)$ \\
\hline 6 a 10 & $191(47,0)$ & $150(78,5)$ & $41(21,5)$ \\
\hline 11 a 14 & $136(33,5)$ & $121(89,0)$ & $15(11,0)$ \\
\hline \multicolumn{4}{|l|}{ Sexo } \\
\hline Masculino & $211(52,0)$ & $170(80,6)$ & $41(19,4)$ \\
\hline Feminino & $195(48,0)$ & $157(80,5)$ & $38(19,5)$ \\
\hline \multicolumn{4}{|c|}{ Autodeclaração de cor da pele ${ }^{a}$} \\
\hline Negro & $145(35,7)$ & $117(80,7)$ & $28(19,3)$ \\
\hline Branco & $12(3,0)$ & $09(75,0)$ & $03(25,0)$ \\
\hline Outros & $247(60,8)$ & $199(80,6)$ & $48(19,4)$ \\
\hline
\end{tabular}

Escolaridade da mãe (anos de estudo) ${ }^{\mathrm{a}}$

\begin{tabular}{|c|c|c|c|}
\hline$<8$ & $201(49,5)$ & $167(83,1)$ & $34(16,9)$ \\
\hline$\geq 8$ & $193(47,5)$ & $155(80,3)$ & $38(19,7)$ \\
\hline \multicolumn{4}{|l|}{ Renda familiar ${ }^{\text {a }}$} \\
\hline$\leq \mathrm{R} \$ 500,00$ & $335(82,5)$ & $274(81,8)$ & $61(18,2)$ \\
\hline$>\mathrm{R} \$ 500,00$ & $63(15,5)$ & $47(74,6)$ & $16(25,4)$ \\
\hline \multicolumn{4}{|c|}{ Tratamento de água a } \\
\hline Sim & $319(78,6)$ & $254(79,6)$ & $65(20,4)$ \\
\hline Não & $87(21,4)$ & $73(83,9)$ & $14(16,1)$ \\
\hline \multicolumn{4}{|c|}{ Forma de tratamento de água } \\
\hline Cloro & $167(41,1)$ & $134(80,2)$ & $33(19,8)$ \\
\hline Filtração & $119(29,3)$ & $101(84,9)$ & $18(15,1)$ \\
\hline Outra & $33(8,1)$ & $19(57,6)$ & $14(42,4)$ \\
\hline $\begin{array}{l}\text { Não trata a } \\
\text { água }\end{array}$ & $87(21,4)$ & $73(83,9)$ & $14(16,1)$ \\
\hline \multicolumn{4}{|c|}{ Peso ao nascer (gramas) ${ }^{\mathrm{a}}$} \\
\hline$<2.500$ & $141(34,7)$ & $111(78,7)$ & $30(21,3)$ \\
\hline 2.500 & $221(54,4)$ & $183(82,8)$ & $38(17,2)$ \\
\hline
\end{tabular}

Intercorrência na gravidez

Sim $289(71,2) \quad 244(84,4) \quad 45(15,6)$

Não $\quad 117(28,8) \quad 83(70,9) \quad 34(29,1)$

Doença na primeira infância

Sim $364(89,7) \quad 298(81,9) \quad 66(18,1)$

Não $\quad 42(10,3) \quad 29(69,0) \quad 13(31,0)$

Frequência de escovação dentária diária ${ }^{a}$

$<3$ vezes $\quad 192(47,3) \quad 151(78,6) \quad 41(21,4)$

$\begin{array}{llll}\geq 3 \text { vezes } \quad 211(52,0) & 173(82,0) & 38(18,0)\end{array}$

Total $406(100,0) \quad 327(80,5) \quad 79(19,5)$

${ }^{\mathrm{a}} \mathrm{Os}$ totais variaram devido à perda de informações.

Fonte: Pesquisa direta, Teresina-PI-Brasil.

\section{Discussão}

Foi observada presença de defeitos de desenvolvimento de esmalte na maioria dos indivíduos, resultados observados em outros estudos com populações de baixa renda ${ }^{10,14,15}$. O diagnóstico precoce de alterações do esmalte dentário apresenta relevância clínica, tendo em vista que DDE predispõe ao acúmulo de biofilme dental que associado à sensibilidade dentária, dificulta a escovação, além de impactar negativamente na qualidade de vida ${ }^{13,17}$.

Insegurança alimentar são fatores associados a DDE, o que justifica a prevalência alta da condição em pessoas que vivem em vulnerabilidade social como é o caso dos quilombolas ${ }^{1,4,5}$, cuja renda familiar média é inferior a um salário mínimo. Íons cálcio presentes na alimentação regulam atividades celulares como comunicação celular, transdução de sinal e ativação enzimática, essenciais para atividade das proteínas envolvidas na amelogênese ${ }^{22}$. Níveis reduzidos de fósforo e cálcio sérico durante a gravidez associados a outras complicações neste período também podem resultar em cristalização e mineralização deficiente do esmalte dentário ${ }^{23}$. A limitação do estudo estão associadas à falta de avaliação da insegurança alimentar por meio de escala válida de monitoração.

Vale enfatizar que deficências em acompanhamentos pré-natais e assistência pediátrica na primeira infância podem aumentar as chances de ocorrência de DDE 9,10,14,15. Tendo em vista que ameloblastos são células sensíveis que podem ser lesadas frente a agressões físico-químicas e biológicas com consequentes danos irreversíveis ao esmalte de dentes em formação $0^{22,24}$.

A prevalência de DDE em dentes decíduos foi menor que em dentes permanentes, resultados que corroboram outros apontados na literatu$\mathrm{ra}^{12,10,15}$. Este resultado pode estar mascarado pela dificuldade de diagnóstico de DDE em dentes decíduos, por apresentarem colorações esbranquiçadas que podem subestimar possíveis defeitos estruturais ${ }^{25}$. Outra justificativa para a baixa prevalência pode estar associada a menor janela de exposição aos fatores ambientais em virtude da calcificação da maioria dos dentes decíduos ocorrer na vida intrauterina, período em que existe a proteção da placenta ${ }^{10,14,15}$.

As áreas dos dentes mais afetadas por DDE foram metade incisal e ponta de cúspide em ambas as dentições, resultado semelhante relatado na literatura ${ }^{26}$. A localização dos defeitos depende da fase do desenvolvimento dentário em que 
Tabela 2. Defeitos de desenvolvimento do esmalte dentário nos dentes decíduos e permanentes.

\begin{tabular}{|c|c|c|}
\hline \multirow{2}{*}{ DDE } & Dentes decíduos & Dentes permanentes \\
\hline & $\mathrm{N}(\%)$ & $\mathbf{N}(\%)$ \\
\hline \multicolumn{3}{|l|}{ Frequência } \\
\hline Sim & $171(42,2)$ & $248(61,1)$ \\
\hline Não & $126(31,0)$ & $85(20,9)$ \\
\hline \multicolumn{3}{|l|}{ Tipos de defeitos } \\
\hline Opacidade demarcada & $83(20,4)$ & $97(23,9)$ \\
\hline Opacidade difusa & $17(4,3)$ & $43(10,6)$ \\
\hline Hipoplasia & $13(3,3)$ & $10(2,5)$ \\
\hline Opacidades demarcada e difusa & $16(3,9)$ & $58(14,3)$ \\
\hline Opacidade demarcada e hipoplasia & $30(7,4)$ & $23(5,7)$ \\
\hline Opacidade difusa e hipoplasia & $03(0,7)$ & $06(1,5)$ \\
\hline Opacidade demarcada, difusa e hipoplasia & $09(2,2)$ & $11(2,7)$ \\
\hline \multicolumn{3}{|l|}{ Localização } \\
\hline Metade gengival & $10(2,5)$ & $50(12,3)$ \\
\hline Metade incisal & $34(8,4)$ & $39(9,7)$ \\
\hline Oclusal & $06(1,5)$ & $03(0,7)$ \\
\hline Cúspide & $61(15,0)$ & $68(16,8)$ \\
\hline Metade gengival e incisal & $08(2,0)$ & $06(1,5)$ \\
\hline Metade gengival e cúspide & $07(1,8)$ & $12(3,0)$ \\
\hline Metade incisal e oclusal & $03(0,7)$ & $05(1,2)$ \\
\hline Metade incisal e cúspide & $19(4,7)$ & $46(11,3)$ \\
\hline Oclusal e cúspide & $03(0,7)$ & $06(1,5)$ \\
\hline Metade gengival, incisal e oclusal & $03(0,7)$ & $01(0,2)$ \\
\hline Metade gengival, incisal e cúspide & $10(2,5)$ & $06(1,5)$ \\
\hline Metade gengival, oclusal e cúspide & $02(0,5)$ & $01(0,2)$ \\
\hline Metade incisal, oclusal e cúspide & $01(0,2)$ & $05(1,2)$ \\
\hline Metade gengival, incisal, oclusal e cúspide & $02(1,0)$ & $00(0,0)$ \\
\hline \multicolumn{3}{|l|}{ Extensão } \\
\hline Menos de $1 / 3$ & $97(23,9)$ & $151(37,2)$ \\
\hline Entre $1 / 3$ e $2 / 3$ & $23(5,7)$ & $04(1,0)$ \\
\hline Mais de $2 / 3$ & $05(1,2)$ & $03(0,7)$ \\
\hline Menos de $1 / 3$ e entre $1 / 3$ e $2 / 3$ & $28(6,9)$ & $49(12,1)$ \\
\hline Menos de $1 / 3$ e mais de $2 / 3$ & $06(1,5)$ & $17(4,2)$ \\
\hline Entre $1 / 3$ e $2 / 3$ e mais de $2 / 3$ & $02(0,5)$ & $03(0,7)$ \\
\hline Menos de $1 / 3$, entre $1 / 3$ e $2 / 3$ e mais de $2 / 3$ & $10(2,5)$ & $21(5,2)$ \\
\hline
\end{tabular}

* Indivíduos com dentes ausentes na dentição decídua $\mathrm{n}=109(26,8 \%) .{ }^{* *}$ Indivíduos com dentes ausentes na dentição permanente $\mathrm{n}$

= 73; Houve associação entre a frequência de DDE na dentição decídua e permanente (teste do Qui-Quadrado de Pearson; $\mathrm{p}=0,018$ ).

Fonte: Pesquisa direta, Teresina-PI-Brasil.

insultos ocorreram e o processo de amelogênese inicia-se nas pontas de cúspides ou borda incisal e progride em direção ao terço cervical do den$\mathrm{te}^{27}$. A maioria de indivíduos apresentou defeitos de esmalte em menos de $1 / 3$ de superfícies dentárias em ambas as dentições, o que corrobora os resultados apresentados por Wagner ${ }^{26}$ e difere dos apontados por Masterson ${ }^{11}$ que afirmou que a maioria dos indivíduos apresentou defeitos de $1 / 3$ a $2 / 3$ da superfície dentária. As características dos defeitos ocorreram precocemente num intervalo de tempo curto.

Opacidades demarcadas foram os defeitos mais frequentes, resultado observado também em outros estudos ${ }^{12,14,26,28}$. Opacidades difusas, como fluorose em graus muito leve e leve, foram mais frequentes que hipoplasias, resultado atribuído possivelmente à ingestão de dentifrício fluoretado no período da amelogênese, em virtude da região não possuir água de abastecimento pú- 
Tabela 3. Associação entre DDE e características socioeconômicas e demográficas, intercorrências durante a gravidez e doença na primeira infância das crianças e adolescentes residentes na comunidade quilombola Lagoas, Sul do Estado do Piauí.

\begin{tabular}{|c|c|c|c|c|c|c|}
\hline \multirow[b]{2}{*}{ Variáveis } & \multicolumn{6}{|c|}{ Defeito de Desenvolvimento do Esmalte } \\
\hline & $\begin{array}{c}\text { Sim } \\
\mathbf{n}(\%)\end{array}$ & $\begin{array}{c}\text { Não } \\
\text { n (\%) }\end{array}$ & $\begin{array}{c}\text { RP não ajustada } \\
\text { (IC 95\%) }\end{array}$ & p-valor ${ }^{b}$ & $\begin{array}{l}\text { RP ajustada } \\
\text { (IC 95\%) }\end{array}$ & p-valor ${ }^{b}$ \\
\hline \multicolumn{7}{|c|}{ Socioeconômicas e demográficas } \\
\hline \multicolumn{7}{|l|}{ Idade (anos) } \\
\hline $3-5$ & $56(17,1)$ & $23(29,1)$ & 1 & & 1 & \\
\hline $6-10$ & $150(45,9)$ & $41(51,9)$ & $1,04(0,98-1,12)$ & 0,201 & $1,03(0,95-1,11)$ & 0,441 \\
\hline $11-14$ & $121(37,0)$ & $15(19,0)$ & $1,11(1,04-1,18)$ & 0,002 & $1,09(1,01-1,17)$ & 0,024 \\
\hline \multicolumn{7}{|l|}{ Sexo } \\
\hline Masculino & $170(52,0)$ & $41(51,9)$ & 1 & & - & - \\
\hline Feminino & $157(48,0)$ & $38(48,1)$ & $1,00(0,96-1,04)$ & 0,987 & - & \\
\hline \multicolumn{7}{|c|}{ Autodeclaração de cor da pele ${ }^{a}$} \\
\hline Negro & $117(36,0)$ & $28(35,4)$ & $1,00(0,96-1,05)$ & 0,976 & - & - \\
\hline Branco & $9(2,8)$ & $3(3,8)$ & $0,97(0,84-1,12)$ & 0,667 & - & - \\
\hline Outros & $199(61,2)$ & $48(60,8)$ & 1 & & - & \\
\hline \multicolumn{7}{|c|}{ Escolaridade da mãe (anos de estudo) ${ }^{\mathrm{a}}$} \\
\hline$<8$ & $167(51,9)$ & $34(47,2)$ & $1,01(0,97-1,05)$ & 0,615 & - & - \\
\hline$\geq 8$ & $155(48,1)$ & $38(52,8)$ & 1 & & - & \\
\hline \multicolumn{7}{|l|}{ Renda familiar $^{\mathrm{a}}$} \\
\hline$\leq \mathrm{R} \$ 500,00$ & $274(85,4)$ & $61(79,2)$ & $1,03(0,97-1,10)$ & 0,328 & - & - \\
\hline$>\mathrm{R} \$ 500,00$ & $47(14,6)$ & $16(20,8)$ & 1 & & - & \\
\hline \multicolumn{7}{|l|}{ Tratamento de água ${ }^{\mathrm{a}}$} \\
\hline Sim & $254(77,7)$ & $65(82,3)$ & 1 & & - & - \\
\hline Não & $73(22,3)$ & $14(17,7)$ & $1,03(0,98-1,08)$ & 0,191 & - & \\
\hline \multicolumn{7}{|c|}{ Peso ao nascer (gramas) ${ }^{a}$} \\
\hline$<2.500$ & $111(37,8)$ & $30(44,1)$ & 1 & & - & - \\
\hline$\geq 2.500$ & $183(62,2)$ & $38(55,9)$ & $1,02(0,98-1,07)$ & 0,342 & - & \\
\hline \multicolumn{7}{|c|}{ Intercorrência na gravidez } \\
\hline \multicolumn{7}{|l|}{ Febre ou infecção ${ }^{a}$} \\
\hline Sim & $60(19,1)$ & $12(16,0)$ & $0,98(0,92-1,04)$ & 0,461 & - & - \\
\hline Não & $254(80,9)$ & $63(84,0)$ & 1 & & - & \\
\hline \multicolumn{7}{|l|}{ Hipertensão $^{\mathrm{a}}$} \\
\hline Sim & $75(23,7)$ & $10(13,5)$ & $1,05(1,00-1,10)$ & 0,048 & - & - \\
\hline Não & $241(76,3)$ & $64(86,5)$ & 1 & & - & \\
\hline \multicolumn{7}{|l|}{ Infecção urinária ${ }^{a}$} \\
\hline Sim & $112(34,5)$ & $23(29,1)$ & $0,99(0,98-1,03)$ & 0,470 & - & - \\
\hline Não & $213(65,5)$ & $56(70,9)$ & 1 & & - & \\
\hline \multicolumn{7}{|l|}{ Uso de antibiótico ${ }^{\mathrm{a}}$} \\
\hline Sim & $151(49,3)$ & $19(27,1)$ & $1,03(0,98-1,09)$ & $<0,001$ & $1,14(1,07-1,22)$ & $<0,001$ \\
\hline Não & $155(50,7)$ & $51(72,9)$ & 1 & & 1 & \\
\hline \multicolumn{7}{|l|}{ Sangramento $^{\mathrm{a}}$} \\
\hline Sim & $20(6,3)$ & $2(2,6)$ & $1,03(0,96-1,11)$ & 0,363 & - & - \\
\hline Não & $298(93,7)$ & $74(97,4)$ & 1 & & - & \\
\hline \multicolumn{7}{|c|}{ Deslocamento prematuro da placenta ${ }^{\mathrm{a}}$} \\
\hline Sim & $22(6,9)$ & $6(7,7)$ & $0,98(0,90-1,08)$ & 0,752 & - & - \\
\hline Não & $298(93,1)$ & $72(92,3)$ & 1 & & - & \\
\hline \multicolumn{7}{|l|}{ Eclâmpsia $^{\mathrm{a}}$} \\
\hline Sim & $2(0,6)$ & $1(1,4)$ & $0,89(0,65-1,23)$ & 0,482 & - & - \\
\hline Não & $310(99,4)$ & $73(98,6)$ & 1 & & - & \\
\hline
\end{tabular}


Tabela 3. Associação entre DDE e características socioeconômicas e demográficas, intercorrências durante a gravidez e doença na primeira infância das crianças e adolescentes residentes na comunidade quilombola Lagoas, Sul do Estado do Piauí.

\begin{tabular}{|c|c|c|c|c|c|c|}
\hline \multirow[b]{2}{*}{ Variáveis } & \multicolumn{6}{|c|}{ Defeito de Desenvolvimento do Esmalte } \\
\hline & $\begin{array}{c}\text { Sim } \\
\mathbf{n}(\%)\end{array}$ & $\begin{array}{l}\text { Não } \\
\text { n (\%) }\end{array}$ & $\begin{array}{c}\text { RP não ajustada } \\
\text { (IC 95\%) }\end{array}$ & p-valor ${ }^{b}$ & $\begin{array}{l}\text { RP ajustada } \\
\text { (IC 95\%) }\end{array}$ & p-valor ${ }^{b}$ \\
\hline \multicolumn{7}{|c|}{ Doença na primeira infância } \\
\hline \multicolumn{7}{|l|}{ Diarréia $^{\mathrm{a}}$} \\
\hline $\operatorname{Sim}$ & $111(35,7)$ & $25(34,2)$ & $0,97(0,92-1,03)$ & 0,351 & - & - \\
\hline Não & $200(64,3)$ & $48(65,8)$ & 1 & & - & \\
\hline \multicolumn{7}{|l|}{ Varicela $^{\mathrm{a}}$} \\
\hline Sim & $59(19,0)$ & $7(9,5)$ & $1,03(0,97-1,10)$ & 0,263 & - & - \\
\hline Não & $252(81,0)$ & $67(90,5)$ & 1 & & - & \\
\hline \multicolumn{7}{|l|}{ Pneumonia $^{\mathrm{a}}$} \\
\hline $\operatorname{Sim}$ & $36(11,4)$ & $10(13,3)$ & $0,97(0,89-1,07)$ & 0,578 & - & - \\
\hline Não & $281(88,6)$ & $65(86,7)$ & 1 & & - & \\
\hline \multicolumn{7}{|l|}{ Asma $^{\mathrm{a}}$} \\
\hline Sim & $37(11,8)$ & $14(18,2)$ & $0,97(0,88-1,06)$ & 0,452 & - & - \\
\hline Não & $277(88,2)$ & $63(81,8)$ & 1 & & - & \\
\hline \multicolumn{7}{|l|}{ Bronquite $^{\mathrm{a}}$} \\
\hline Sim & $17(5,4)$ & $5(6,8)$ & $0,98(0,87-1,11)$ & 0,793 & - & - \\
\hline Não & $295(94,6)$ & $68(93,2)$ & 1 & & - & \\
\hline \multicolumn{7}{|l|}{ Sinusite ${ }^{\mathrm{a}}$} \\
\hline Sim & $26(8,6)$ & $4(5,6)$ & $1,04(0,96-1,12)$ & 0,313 & - & - \\
\hline Não & $277(91,4)$ & $68(94,4)$ & 1 & & - & \\
\hline \multicolumn{7}{|l|}{ Rinite $^{\mathrm{a}}$} \\
\hline Sim & $31(9,8)$ & $4(5,3)$ & $1,01(0,92-1,10)$ & 0,855 & - & - \\
\hline Não & $286(90,2)$ & $71(94,7)$ & 1 & & - & \\
\hline \multicolumn{7}{|l|}{ Febre $^{\mathrm{a}}$} \\
\hline $\operatorname{Sim}$ & $152(49,0)$ & $25(34,2)$ & $1,02(0,96-1,08)$ & 0,490 & - & - \\
\hline Não & $158(51,0)$ & $48(65,8)$ & 1 & & - & \\
\hline \multicolumn{7}{|c|}{ Relato de desnutrição ${ }^{a}$} \\
\hline Sim & $44(14,0)$ & $4(5,3)$ & $1,13(1,04-1,22)$ & 0,003 & $1,12(1,03-1,22)$ & 0,011 \\
\hline Não & $270(86,0)$ & $71(94,7)$ & 1 & & 1 & \\
\hline \multicolumn{7}{|l|}{ Otite $^{\mathrm{a}}$} \\
\hline $\operatorname{Sim}$ & $68(21,2)$ & $11(15,1)$ & $0,99(0,92-1,07)$ & 0,887 & - & - \\
\hline Não & $252(78,8)$ & $62(84,9)$ & 1 & & - & \\
\hline \multicolumn{7}{|l|}{ Uso de Antibiótico ${ }^{\mathrm{a}}$} \\
\hline Sim & $154(51,0)$ & $33(47,1)$ & $0,98(0,93-1,04)$ & 0,510 & - & - \\
\hline Não & $148(49,0)$ & $37(52,9)$ & 1 & & - & \\
\hline
\end{tabular}

blico fluoretada e as crianças e adolescentes terem nascido e residido sempre nas comunidades ${ }^{29}$. O diagnóstico de fluorose foi realizado por meio do índice DDE modificado ${ }^{21}$. Crianças cujos pais são orientados sobre a quantidade de dentifrício fluoretado a ser utilizada desde os primeiros anos de vida apresentaram menor frequência com fluorose dentária nos dentes permanentes ${ }^{30}$. No presente estudo, a prevalência alta de fluorose na dentição permanente pode ser atribuída a ingestão de dentifrícios fluoretados durante o período de calcificação das coroas dentárias, tendo em vista que os participantes do estudo nasceram e moram nas comunidades quilombolas, sem nunca terem se ausentado por períodos mais longos que 30 dias. 
Neste estudo, foi observada maior frequência de DDE nas faixas etárias de 6 a 10 anos e de 11 a 14 anos. De fato, a amelogênese é um processo longo que inicia na vida intrauterina e se prolonga até a primeira infância então, perturbações durante esse processo poderá ser fator desencadeante de DDE tanto em dentes decíduos quanto em permanentes, dependendo do momento em que ocorreu o insulto ${ }^{28}$. A calcificação da maioria dos dentes permanentes ocorre na primeira infância e nesta fase do crescimento e desenvolvimento existe maior chance da ocorrência de doenças com comprometimento sistêmico que provocam febres altas e são associadas a $\mathrm{DDE}^{14}$.

Complicações durante a gestação ${ }^{27}$, condições adversas ao nascimento ${ }^{28}$, deficiências nutricionais ${ }^{7}$ e doenças na primeira infância têm sido associadas com o aumento da frequência de DDE, e a justificativa é a associação com a função ameloblástica ${ }^{6}$. No presente estudo, o uso de medicamentos na gravidez foi associado à presença de
DDE, e antibióticos os mais referidos pelas mães, resultado concordante com a literatura ${ }^{20}$. No entanto, é importante considerar o viés de memória como limitante em estudos transversais. Para facilitar o entendimento do questionário, houve treinamento prévio dos pesquisadores visando facilitar o entendimento de termos técnicos em linguagem acessível.

Este estudo contempla um grupo prioritário de pesquisa em saúde no Brasil e deperta a atenção para a associação estabelecida de DDE com fatores sociais e doenças bucais. Possibilita, assim, planejamentos de estratégias capazes de prevenir problemas de saúde, uma vez que muitos programas são centrados historicamente no tratamento de condições já estabelecidas.

Desta forma, a prevalência de DDE em crianças e adolecentes da comunidade quilombola foi alta. Os fatores associados foram maior idade da criança, uso de antibióticos na gravidez e desnutrição durante a primeira infância.

\section{Colaboradores}

TKXS França colaborou na concepção do projeto, coleta de dados, avaliação dos resultados, escrita do artigo, revisão do artigo e resposta aos revisores. MDM Lima colaborou na concepção do projeto, coleta de dados, avaliação dos resultados, revisão crítica do artigo e resposta aos revisores. CCB Lima fez análise estatística, avaliação dos resultados, revisão crítica do artigo e resposta aos revisores. MS Moura colaborou na coleta de dados, avaliação dos resultados, revisão crítica do artigo e resposta aos revisores. TSP Lopes fez coleta de dados, avaliação dos resultados, revisão crítica do artigo e resposta aos revisores. JSS Moura colaborou na coleta de dados, avaliação crítica dos resultados, escrita do artigo. LFAD Moura colaborou na concepção e orientação do projeto, coleta de dados, avaliação crítica dos resultados, escrita do artigo e revisão crítica do artigo, submissão e resposta aos revisores. 


\section{Referências}

1. Silva EKPD, Medeiros DS, Martins PC, Sousa LA, Lima GP, Rêgo MAS, Silva TOD, Freire AS, Silva FM. Insegurança alimentar em comunidades rurais no Nordeste brasileiro: faz diferença ser quilombola? Cad Saude Publica 2017; 33(4):e00005716.

2. Souza MCA, Flório FM. Avaliação da história da cárie e fatores associados entre os quilombolas no Sudeste do Brasil. Braz J Ora Sci 2014; 13(3):175-181.

3. Bidinotto $A B$, D'Ávila OP, Martins $A B$, Hugo FN, Neutzling MB, Bairros FS, Hilgert JB. Oral health self-perception in quilombola communities in Rio Grande do Sul: a cross-sectional exploratory study. Rev Bras Epidemiol 2017; 20(1):91-101.

4. Marques AS, Freitas DA, Leão CD, Oliveira SK, Pereira MM, Caldeira AP. Atenção Primária e saúde materno-infantil: a percepção de cuidadores em uma comunidade rural quilombola. Cien Saude Colet 2014; 19(2):365-371.

5. Gubert MB, Spaniol AM, Bortolini GA, Pérez-Escamilla R. Household food insecurity nutritional status and morbidity in Brazilian children. Public Health Nutr 2016; 19(12):2240-2245.

6. Robles MJ, Ruiz M, Bravo-Perez M, González E, Peñalver MA. Prevalence of enamel defects in primary and permanent teeth in a group of schoolchildren from Granada. Med Oral Patol Oral Cir Bucal 2013;18(2):187-193.

7. Yadav PK. Prevalence and Association of Developmental Defects of Enamel with, Dental-Caries and Nutritional Status in Pre-School Children, Lucknow. J Clin Diagn Res 2015; 9(10):71-74.

8. Ford D, Seow WK, Kazoullis S, Holcome T, Newman B. A controlled study of risk factors for enamel hypoplasia in the permanent dentition. Pediatr Dent 2009; 31(5):382-388.

9. Massoni AC, Chaves AM, Sampaio FC, Rosenblatt A; Oliveira AF. Prevalence of enamel defects related to pre-, peri- and postnatal factors in a Brazilian population. Community Dent Health 2009; 26(3):143-149.

10. Basha S, Mohamed RN, Swamy HS. Prevalence and associated factors to developmental defects of enamel in primary and permanent dentition. Oral Health Dent Manag 2014; 13(3):588-594.

11. Masterson EE, Fitzpatrick AL, Enquobahrie DA, Mancl LA, Conde E, Hujoel PP. Malnutrition-related early chidhood exposures and enamel defects in the permanente dentition: a longitudinal study from the Bolivian Amazon. Am J Phys Anthropol 2017; 164(2):416423

12. Jacobsen PE, Haubek D, Henriksen TM, Østergaard JR, Poulsen S. Developmental defects of enamel in children born preterm: a systematic review. Eur J Oral Sci 2014; 122(1):7-14.

13. Mermarpour M, Golkari A, Ahmadian R. Association of characteristics of delivery and medical conditions during the first month of life with developmental defects of enamel. BMC Oral Health 2014; 14:122.

14. Hoffmann RHS, Sousa MDLR, Cypriano S. Prevalência de defeitos de esmalte e sua relação com cárie dentária nas dentições decídua e permanente, Indaiatuba, São Paulo, Brasil. Cad Saude Publica 2007; 23(2):435444.
15. Vargas-Ferreira F, Ardenghi TM. Developmental enamel defects and their impact on child oral health-related quality of life. Braz Oral Res 2011; 25(6):531-537.

16. Salas MM, Nascimento GG, Tarquinio SB, Faggion CM Jr, Peres MA, Thomson WM, Demarco FF. Association between developmental defects of enamel and dental caries: A systematic review and meta-analysis. J Dent 2015; 43(6):619-628.

17. Costa FS, Silveira ER, Pinto GS, Nascimento GG, Thomson WM, Demarco F. Developmental defects of enamel and dental caries in the primary dentition: a systematic review and meta-analysis. J Dent 2017; 60:1-7.

18. Instituto Nacional de Colonização e Reforma Agrária (INCRA). Relatório Antropológico do Território Quilombola de Lagoas. Brasília: INCRA; 2010.

19. Brasil. Ministério da Saúde (MS). Coordenação Nacional de Saúde Bucal. Projeto SBBrasil 2010 - Pesquisa Nacional de Saúde Bucal: manual da Equipe de Campo. Brasília: MS; 2009.

20. Ravindran R, Saji AM. Prevalence of the developmental defects of the enamel in children aged 12-15 years in Kollam district. J Int Soc Prev Community Dent 2016; 6 (1):28-33.

21. Federation Dental International. A review of the developmental defects of enamel index (DDE Index). Commission on Oral Health, Research \& Epidemiology. Report of an FDI Working Group. Int Dent J 1992; 42(6):411-412.

22. Simmer JP, Hu JC. Expression, structure, and function of enamel proteinases. Connect Tissue Res 2002; 43(23):441-449.

23. Merheb R, Arumugam C, Lee W, Collin M, Nguyen C, Groh-Wargo S, Nelson S. Neonatal serum phosphorus levels and enamel defects in very-low-birth -weight infants. JPEN J Parenter Enteral Nutr 2016; 40(6):835-841.

24. Brook AH. Multilevel complex interactions between genetic, epigenetic and enviromental factors in the aetiology of anomalies of dental development. Arch Oral Biol 2009; 54(1):S3-17.

25. Masumo R, Bardsen A, Astrøm AN. Developmental defects of enamel in primary teeth and association with early life course events: a study of 6-36 month old children in Manyara, Tanzania. BMC Oral Health 2013; 13:21.

26. Wagner Y. Developmental defects of enamel in primary teeth - findings of a regional German birth cohort study. BMC Oral Health 2016; 17(1):10.

27. Velló MA, Martínez-Costa C, Catalá M, Fons J, Brines J, Guiiarro-Martínez R. Prenatal and neonatal risk factors for the development of enamel defects in low birth weight children. Oral Dis 2010; 16(3):257-262.

28. Correa-Faria P, Martins-Junior PA, Vieira-Andrade RG, Marques LS, Ramos-Jorge ML. Perinatal factors associated with developmental defects of enamel in primary teeth: a case-control study. Braz Oral Res 2013; 27(4):363-368. 
29. Goodarzi F, Mahvi AH, Hosseini M, Nedjat S, Nabizadeh Nodehi R, Kharazifard MJ, Parvizishad M, Cheraghi Z. The prevalence of dental fluorosis and exposure to fluoride in drinking water: A systematic review. $J$ Dent Res Dent Clin Prospects 2016; 10(3):127-135.

30. Moura MS, Carvalho MM, Silva MC, Lima MDM, Moura LFAD, Simplício AHM.The impact of a dental program for maternal and infant health on the prevalence of dental fluorosis. Pediatr Dent 2013; 35(7):519-522.

Artigo apresentado em 04/03/2019

Aprovado em 02/08/2019

Versão final apresentada em 04/08/2019

Editores-chefes: Romeu Gomes, Antônio Augusto Moura da Silva 